APS/123-QED

\title{
Role of Noise in a Market Model with Stochastic Volatility
}

\author{
Giovanni Bonanno, Davide Valenti ${ }^{\circ}$ and Bernardo Spagnolo ${ }^{\dagger}$ \\ Dipartimento di Fisica e Tecnologie Relative, \\ Group of Interdisciplinary Physics *, Università di Palermo \\ Viale delle Scienze, I-90128 Palermo, Italy \\ -valentid@gip.dft.unipa.it, ${ }^{\dagger}$ spagnolo@unipa.it
}

(Dated: November 15, 2018)

\begin{abstract}
We study a generalization of the Heston model, which consists of two coupled stochastic differential equations, one for the stock price and the other one for the volatility. We consider a cubic nonlinearity in the first equation and a correlation between the two Wiener processes, which model the two white noise sources. This model can be useful to describe the market dynamics characterized by different regimes corresponding to normal and extreme days. We analyze the effect of the noise on the statistical properties of the escape time with reference to the noise enhanced stability (NES) phenomenon, that is the noise induced enhancement of the lifetime of a metastable state. We observe NES effect in our model with stochastic volatility. We investigate the role of the correlation between the two noise sources on the NES effect.

PACS numbers: 89.65.Gh; 02.50.-r; 05.40.-a; 89.75.-k
\end{abstract}

Keywords: Econophysics, Stock market model, Langevin-type equation, Heston model, Complex Systems

\footnotetext{
* Electronic address: http://gip.dft.unipa.it
} 


\section{INTRODUCTION}

The presence of noise in physical systems is a well known phenomenon. It is common opinion that noise affecting the dynamics of a system introduces some degree of instability to the system itself, but there is evidence that in some cases the noise can increase the stability of a system [1, 2, 3, 4]. Noise modeling through the use of the stochastic processes formalism has applications that involve many systems including physics, biology, ecology [5, 6, 6] and even financial markets [8, 9, 10]. The most basic model for financial market is the geometric Brownian motion [12]. This model has different drawbacks, it cannot reproduce in fact three important stylized facts observed in financial time series: (i) the non Gaussian distribution of returns, (ii) the fat tails [9, 10], and (iii) the stochastic character of volatility, which is characterized by long range memory and clustering [9, 10, 11, 13]. More complex models have been developed to reproduce the dynamics of the volatility. It is worthwhile citing the ARCH [14] and GARCH [15] models, where the actual volatility depends on the past values of squared return $(\mathrm{ARCH})$ and also on the past values of the volatility (GARCH). Another class of models use a system of stochastic equations writing the price as a geometric Brownian motion coupled with a non-constant volatility described by a second stochastic differential equation. The Heston model uses for the volatility a multiplicative stochastic process characterized by mean reversion [16, 17]. Both the models presented so far have exponential autocorrelation function so they are not able to reproduce quantitatively the long range memory observed in real markets. Nonetheless using values of the characteristic time that are sufficiently high, they are able to give accurate statistic for the stock prices, by tuning only few parameters. Another important characteristic of financial markets is the presence of different regimes. Markets indeed present days of normal activity and extreme days where very high or very low price variations can be observed. These are known as

crash and rally days. A nonlinear Langevin market model has already been proposed [18], where different regimes are modelled by means of an effective potential for price returns. In some circumstances this potential has a cubic shape with a metastable state and a potential barrier. The dynamics inside the metastable state represents the days of normal evolution while the escape after the potential barrier represents the beginning of a crisis. Metastable states are ubiquitous in physics and the effect of noise in such systems has been extensively studied [1, 2, 3, 4], but considering the noise intensity as a parameter (physical models 
described by additive stochastic differential equations for example). Financial markets with their stochastic volatility are an example of systems where the noise intensity is far from being a constant parameter, but it is indeed a stochastic process itself. Moreover there is evidence in nature that the noise intensity is not a constant parameter and can be modelled as a multiplicative noise [6, 7, 19]. So it is interesting to release the hypothesis of parametric noise intensity in financial market models as well as in natural systems.

\section{THE HESTON MODEL WITH A METASTABLE STATE}

The Heston model introduced in the previous section is described by the following system of coupled stochastic differential equations [16]

$$
\begin{aligned}
& d x(t)=(\mu-v(t) / 2) \cdot d t+\sqrt{v(t)} \cdot d Z(t) \\
& d v(t)=a(b-v(t)) \cdot d t+c \sqrt{v(t)} \cdot d W(t)
\end{aligned}
$$

The price $p(t)$ follows a geometric random walk whose standard deviation is another stochastic process. Here $x(t)=\ln p(t)$ is the $\log$ of the price, $Z(t)$ and $W(t)$ are uncorrelated Wiener processes with the usual statistical properties: (i) $\langle d Z(t)\rangle=0$ and $\left\langle d Z(t) \cdot d Z\left(t^{\prime}\right)\right\rangle=\delta\left(t-t^{\prime}\right) d t$; (ii) $\langle d W(t)\rangle=0$ and $\left\langle d W(t) \cdot d W\left(t^{\prime}\right)\right\rangle=\delta\left(t-t^{\prime}\right) d t$. The $v(t)$ process is characterized by mean reversion, i.e. its deterministic solution has an ex-

ponential transient with characteristic time equal to $a^{-1}$, after which the process tends to its asymptotic value $b$. The process for $v(t)$ exhibits the phenomenon of volatility clustering, alternating calm with burst periods of volatility, and has an exponential autocorrelation function. The smaller the value of $a$ the longer are the bursts in volatility. Heston model has been subject of recent investigation by econophysicists [20, 21, 22, 23]. The equations of the system (1) are well known in finance, they represent respectively the log-normal geometric Brownian motion stock process used by Black and Scholes for option pricing [24, 25], and the Cox-Ingersoll-Ross (CIR) mean-reverting stochastic differential equation first introduced for interest rate models [26, 27].

Here we consider a generalization of the Heston model, by replacing the geometric Brownian motion with a random walk in the presence of a cubic nonlinearity. This generalization represents a "Brownian particle" moving in an effective potential with a metastable state, 
in order to model those systems with two different dynamical regimes like financial markets in normal activity and extreme days [18]. The equations of the new model are

$$
\begin{aligned}
& d x(t)=-\left(\frac{\partial U}{\partial x}+\frac{v(t)}{2}\right) \cdot d t+\sqrt{v(t)} \cdot d Z(t) \\
& d v(t)=a(b-v(t)) \cdot d t+c \sqrt{v(t)} \cdot d W(t),
\end{aligned}
$$

where $U$ is the effective cubic potential $U(x)=p x^{3}+q x^{2}$, with $p=2$ and $q=3$ (see Fig. (1)),

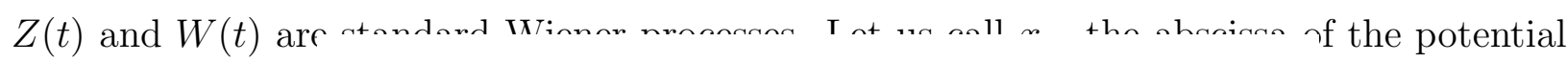

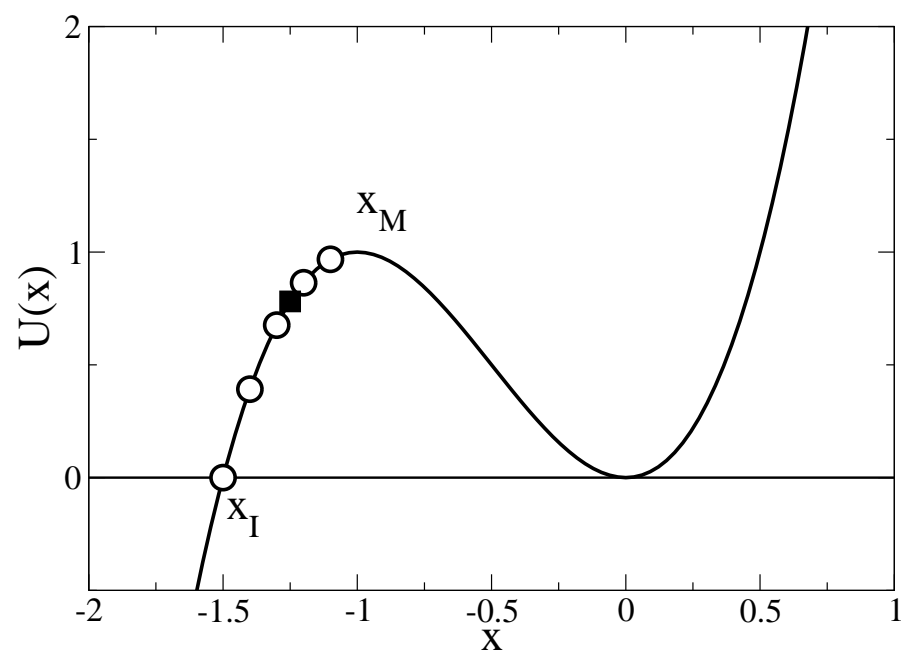

FIG. 1: Cubic potential used in the dynamical equation for the price $\mathrm{x}(\mathrm{t})$. The points in the figure indicate the starting positions used in our simulations.

maximum and $x_{I}$ the abscissa where the potential intersects the $x$ axes. The intervals $x_{o}<x_{I}$ and $I=\left[x_{I}, x_{M}\right]$ are clearly regions of instability for the system. In systems with a metastable state like this, the noise can originate interesting effects that increase instead of decrease the stability, by enhancing the lifetime of the metastable state [1, 2, 3, 4]. A good example is the Noise Enhanced Stability (NES) phenomenon. The mean escape time $\tau$ for a Brownian particle moving throughout a barrier $\Delta U$, with a noise intensity $v$, is given by the well known exponential Kramers law [28, 29]

$$
\tau=A \exp \left[\frac{\Delta U}{v}\right],
$$

where $\tau$ is a monotonically decreasing function of the noise intensity $v$, and $A$ is a prefactor which depends on the potential profile. This is true only if the random walk starts from 
initial positions inside the potential well. When the starting position is chosen in the instability region $x_{o}<x_{M}, \tau$ exhibits an enhancement behavior, with respect to the deterministic escape time, as a function of $v$. Particularly for initial positions $x_{0}<x_{I}$, we have nonmonotonic behavior of $\tau$ as a function of $v$ 22, 30]. This is the NES effect and can be explained considering the barrier "seen" by the Brownian particle starting at the initial position $x_{0}$, that is $\Delta U_{\text {in }}=U\left(x_{\max }\right)-U\left(x_{0}\right)$. Moreover $\Delta U_{\text {in }}$ is less than $\Delta U$ as long as the starting position $x_{0}$ lyes into the interval $I=\left[x_{I}, x_{M}\right]$. Therefore for a Brownian particle, from a probabilistic point of view, it is easier to enter into the well than to escape from, when the particle is entered. So a small amount of noise can increase the lifetime of the metastable state [1, 2, 4, 30]. For a detailed discussion on this point and different dynamical regimes see Refs. [2, 30]. When $v$ is much greater than $\Delta U$, the Kramers behavior is recovered. The NES effect has been experimentally observed in a tunnel diode and theoretically predicted in a wide variety of systems such as for example chaotic map, Josephson junctions, chemical reaction kinetics, and neuronal dynamics models [1, 2, 3, 4]. Our modified Heston model, characterized by a stochastic volatility and a nonlinear Langevin equation for the returns, has two limit regimes, corresponding to the cases $a=0$, with only the noise term in the equation for the volatility $v(t)$, and $c=0$ with only the reverting term in the same equation. This last case corresponds to the usual parametric constant volatility case. In fact, apart from an exponential transient, the volatility reaches the asymptotic value $b$. The NES effect should be observable in the latter case as a function of $b$, which is the average volatility. In this case, in fact, we have the motion of a Brownian particle in a fixed cubic potential with a metastable state and an enhancement of its lifetime for particular initial conditions (see Refs. [2, 30] ).

\section{ENHANCEMENT OF THE ESCAPE TIME}

The two processes of Eqs. (11) and (2) are actually uncorrelated. In financial markets the two processes can be correlated, and a negative correlation between the processes is known

as leverage effect 31|. Heston model with correlation has been recently discussed in the scientific literature [21, 23]. Our modified Heston model becomes therefore 


$$
\begin{aligned}
d x(t) & =-\left(\frac{\partial U}{\partial x}+\frac{v(t)}{2}\right) \cdot d t+\sqrt{v(t)} \cdot d Z(t) \\
d v(t) & =a(b-v(t)) \cdot d t+c \sqrt{v(t)} \cdot d W_{c}(t) \\
d W_{c}(t) & =\rho \cdot d Z(t)+\sqrt{1-\rho^{2}} \cdot d W(t),
\end{aligned}
$$

where $Z(t)$ and $W(t)$ are uncorrelated Wiener processes as in Eqs. (11) and (2), and $\rho$ is the cross correlation coefficient between the noise sources. The investigation is performed simulating the process of Eqs. (41) with time integration step $\Delta t=0.01$, and for the fixed starting position $x_{0}=-1.25$ in the $I$ interval (this initial position is shown as a black square point in Fig. (1). The absorbing barrier is located at $x=-6.0$, and the results are averaged over $10^{5}$ escape events. The algorithm used to simulate the noise sources in Eqs. (4) is based on the Box-Muller method for generating random processes with a Gaussian distribution. The numerical integration of Eqs. (44) is done by using the forward Euler method [32].

Our first result shows that the curve $\tau$ vs. $b$ is weakly dependent on the value of the $\rho$ parameter. This is shown in Fig. 2, where all the curves correspond to the region of the parameters space where the effect is observable. There is indeed a weak variation in the maximum value. The highest maximum values correspond to the highest absolute $\rho$ values. It is worth noting that the correlation affects directly the noise term in the $v(t)$ equation, and it has negligible influence on the reverting term of the same equation.

The curves of $\tau$ vs. $c$ however have an evident dependence on the parameter $c$. This is shown in Fig. 3, for two values of parameter $a$ for which the NES effect is observable. The correlation affects the position as well as the value of the maximum of $\tau$ (see Fig. [3), but in a different way. The position of the maximum increases only for very high positive values of the correlation coefficient. The maximum value of $\tau$ increases with positive correlation but decreases with negative correlation. This effect is more evident for the higher value of $a$, as shown in Fig. 3b. To comment this result we first note that a negative correlation between the logarithm of the price and the volatility means that a decrease in $x(t)$ induces an increase in the volatility $v(t)$, and this causes the Brownian particle to escape easily from the well. As a consequence the mean lifetime of the metastable state decreases, even if the nonmonotonic behavior is still observable. On the contrary, when the correlation $\rho$ is positive, the Brownian particle stays more inside the well, decrease in $x(t)$ indeed is associated with decrease in the volatility. The escape process becomes slow and this increases further the lifetime of the 


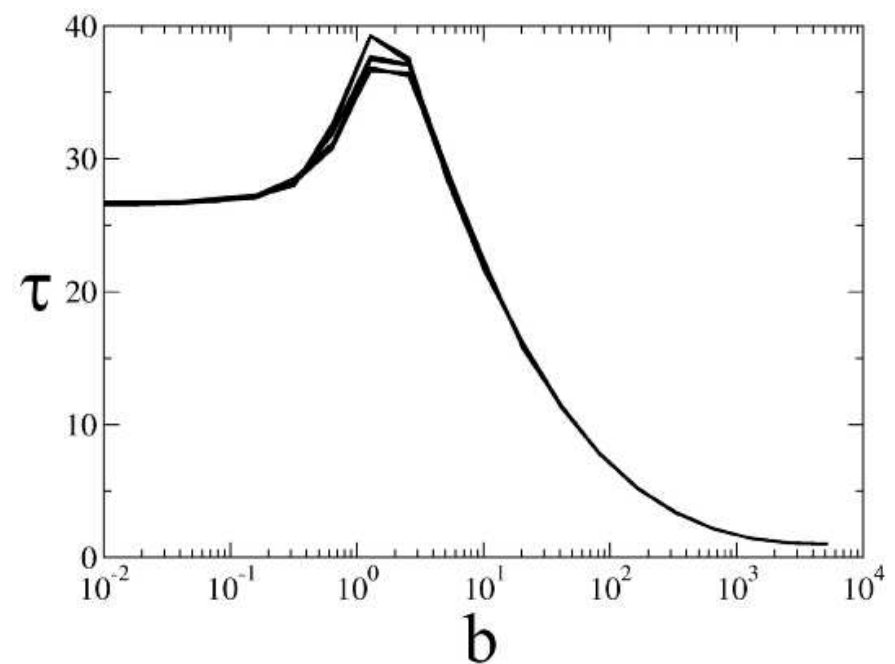

FIG. 2: Mean escape time $\tau$ as a function of the mean volatility $b$. The other parameters have the fixed values $a=10^{-2}$ and $c=10^{-2}$. The six curves correspond to the following values of the correlation: $\rho=-0.8,-0.5,-0.1,0.1,0.5,0.8$. The fixed starting position is $x_{0}=-1.25$. The values of the potential parameters (see Eq. (2)) are: $p=2, q=3$.

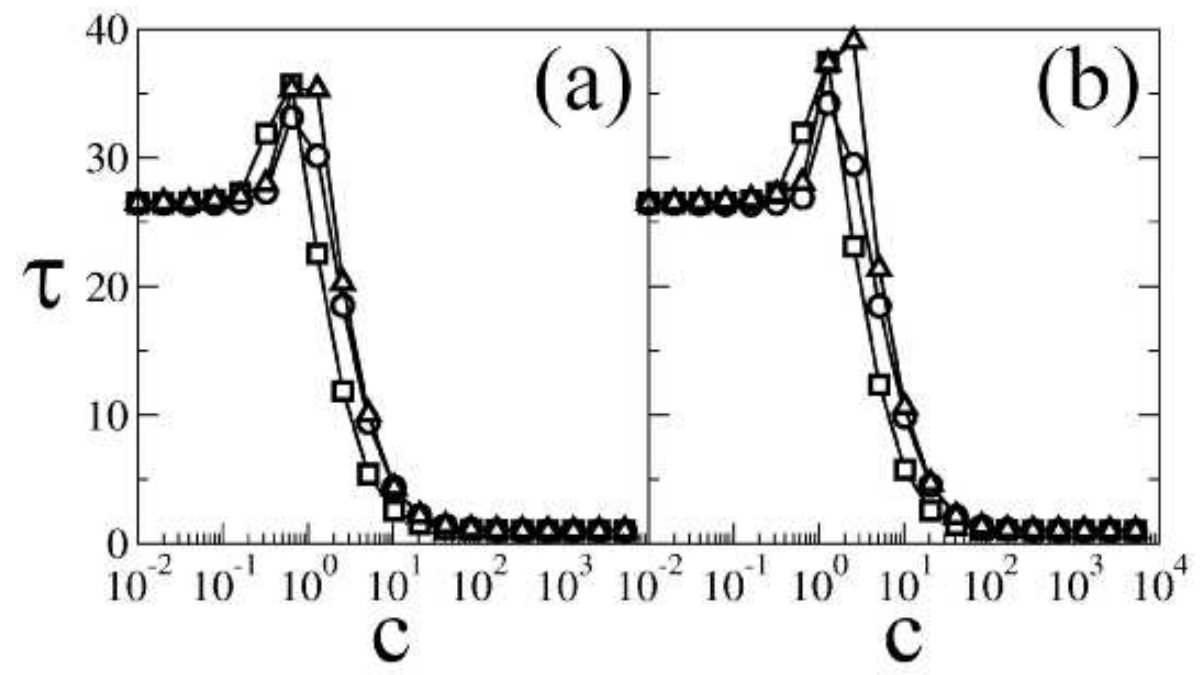

FIG. 3: Mean escape time $\tau$ as a function of the model noise intensity $c$ for two different values of the parameter $a$ : (a) $a=2.0$ and (b) $a=20$. The value of the parameter $b$ is fixed to $b=10^{-2}$. The fixed starting position $x_{0}$ and the potential parameters $p$ and $q$ are the same of Fig. 2. The different curves correspond to the following values of $\rho$ : -0.8 (circle), 0.0 (square), 0.8 (triangle). 
metastable state, causing an increase in the value of the maximum of the curve of Fig. 3. To illustrate better this aspect and the behaviour of Figs. 2 and 3 near the maximum, we plot the values $\tau_{\max }$ of the maximum as a function of the correlation coefficient $\rho$ and we show these curves in Fig. 4. Specifically in Fig. 27 we report the values of $\tau_{\text {max }}$ related to the curves of $\tau$ of Fig. 2, and in Fig. 2 $\mathrm{b}$ those related to Fig. 3. The increase of stability is

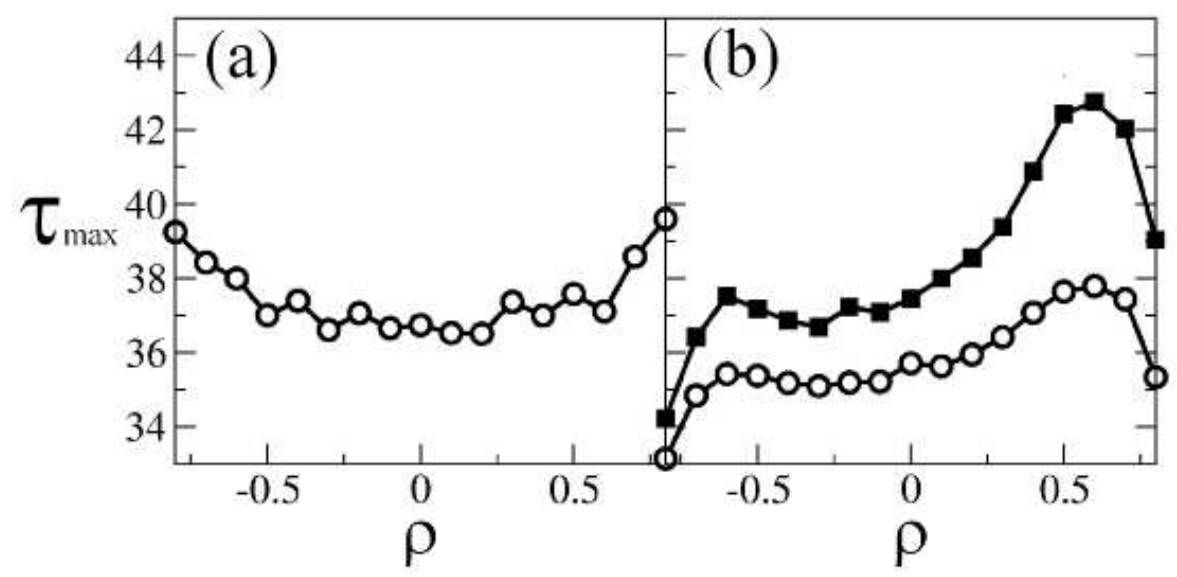

FIG. 4: The values of $\tau_{\max }$ as a function of the correlation coefficient of the curves reported in Figs. 2] and 3. Specifically: (a) $\tau_{\max }$ from Fig. 2 ( $\tau$ as a function of parameter b) with $a=10^{-2}$, $c=10^{-2}$ ); (b) $\tau_{\max }$ from Fig. 3 with $a=2.0$ (circles) and $a=20$ (squares), $b=10^{-2}$. The fixed starting position $x_{0}$ and the potential parameters $p$ and $q$ are the same of Fig. 2 ,

evident in panel (b) as $\tau_{\max }$ increases with $\rho$.

It is interesting to show the probability density function (PDF) of stock price returns for the model described by the Eqs. (4). This is done in Fig. 5. As one can see the qualitative behavior of a fat tail distribution, typical of real financial market data, is recovered [9, 10], but with a peculiar asymmetry. To characterize quantitatively the PDF of returns (Fig. 51) as regards the width, the asymmetry and the fatness of the distribution, we calculate the mean value $\langle\Delta x\rangle$, the variance $\sigma_{\Delta x}$, the skewness $\kappa_{3}$ and the kurtosis $\kappa_{4}$. We obtained the following values: $\langle\Delta x\rangle=-0.434, \sigma_{\Delta x}=0.903, \kappa_{3}=-2.086, \kappa_{4}=9.417$. These statistical quantities clearly show the asymmetry of the distribution and its leptokurtic nature observed in empirical investigations, characterized by a narrow and larger maximum, and by fatter tails than in the Gaussian distribution [9, 10].

The presence of the asymmetry is very interesting and it will be subject of future inves- 


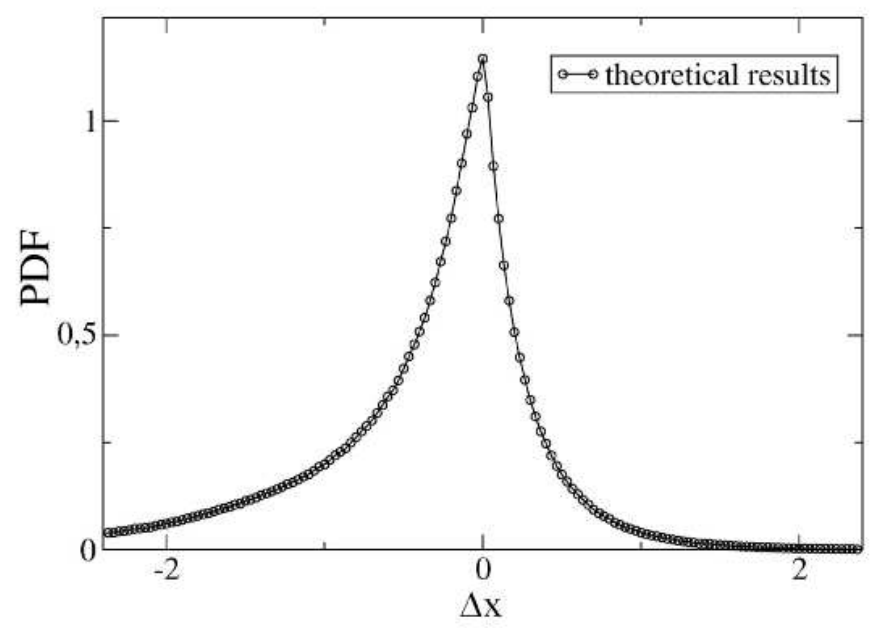

FIG. 5: Probability density function of stock price returns for the Heston model with a metastable state and correlated noise sources (Eqs. (44)). The parameters of the Cox-Ingersoll-Ross process of Eqs. (41) are: $a=2, b=0.01, c=26$. The cross correlation coefficient is $\rho=-0.3$. The potential parameters are the same of Fig. 2

tigations. It is worthwhile to note, however, that the PDF of returns become asymmetric in crash and rally days [33, 34], that are just the time periods of financial data where the related dynamical regimes could be described by the models (2) and (4). Of course the quantitative agreement between the PDF of real data and that obtained from these models requires further investigations on the parameter value choice of the cubic potential, the parameters of the simple Heston model and the correlation coefficient. This analysis is outside the aim of the present work and will be further investigated in a forthcoming paper.

Finally in the following Fig. 6] we report the comparison between the probability density function of the escape times of daily price returns from real market data and that obtained from the model described by the Eqs. (44). The data set used here consists of daily closure prices for 1071 stocks traded at the NYSE and continuously present in the 12-year period 1987 - 1998 (3030 trading days). The same data were used in previous investigations by one of the authors [20, 35, 36]. From this data set we obtained the time series of the returns and we calculated the time to hit a fixed threshold starting from a fixed initial position. The two thresholds were chosen as a fraction of the standard deviation $\sigma_{n}$ observed for each stock during the above mentioned whole time period ( $\mathrm{n}$ is the stock index, varying between 1 and 1071). Specifically we chose: $\left(\Delta x_{i}\right)_{n}=-0.1 \sigma_{n}$ and $\left(\Delta x_{f}\right)_{n}=-1.0 \sigma_{n}$. The 
parameters of the CIR process are: $a=20, b=0.01, c=2.4$. The cross correlation coefficient is $\rho=-0.9$, and the potential parameters are the same of Fig. 2. As one can see the agreement between real and theoretical data is very good, except at small escape times. The choice of this parameter data set is not based on a fitting procedure as that used for example in Ref. 21], where the minimization of the mean square deviation between the PDF of the returns extracted from financial data and that obtained theoretically is done. We chose the parameter set in the range in which we observe the nonmonotonic behaviour of the mean escape time as a function of the parameters $b$ and $c$. Then by a trial and error procedure we selected the values of the parameters $a, b$, and $c$ for which we obtain the best fitting between the PDF of escape times of the price returns calculated from the modified Heston model (Eqs. (44)) and that obtained from time series of real market data. Of course a better quantitative procedure could be done, by considering also the potential parameters. This will be done, together with a detailed analysis of PDF of returns and its asymmetry, in a forthcoming paner

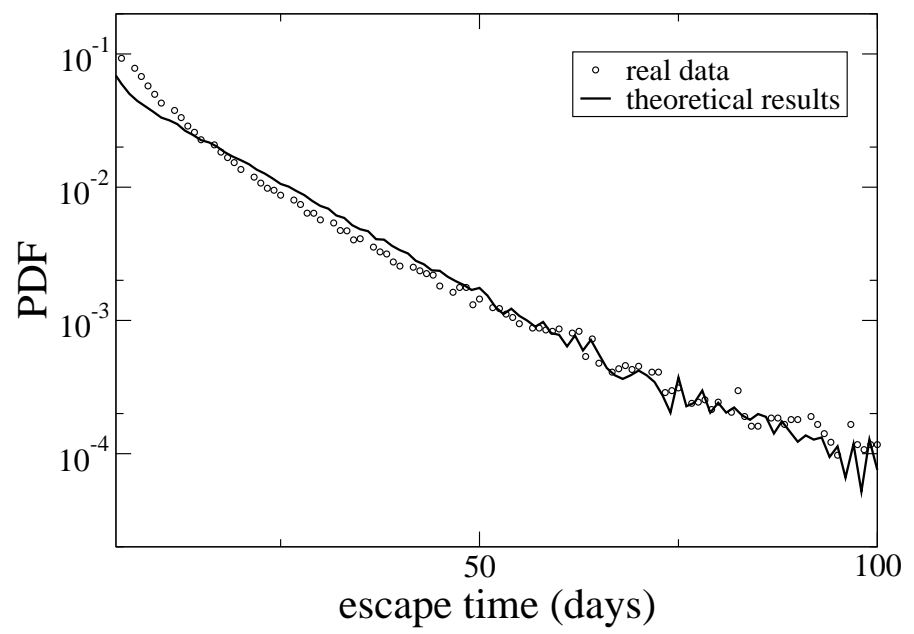

FIG. 6: Comparison between the probability density function of escape times of the price returns for the modified Heston model with a metastable state (Eqs. (4)) (solid line) and the PDF of escape times of returns for real market data (cirles). The parameter values of the model (44) are: $p=2$, $q=3, a=20, b=10^{-2}$, and $c=2.4$. The cross correlation coefficient between the noise sources is: $\rho=-0.9$. 


\section{CONCLUSIONS}

We have investigated the statistical properties of the escape time in a generalized Heston market model, characterized by the presence of a metastable state in the effective potential of the logarithm of the price $p(t)$. We observe the NES effect in the system investigated. The presence of correlation between the stochastic volatility and the noise source which affects directly the dynamics of the quantity $x(t)=\ln p(t)$ (as in usual market models), can

influence the stability of the market. Specifically a positive correlation between $x(t)$ and volatility $v(t)$ slows down the walker escape process, that is it delays the crash phenomenon by increasing the stability of the market. A negative correlation on the contrary accelerates the escape process, lowering the stability of the system.

This work was partially supported by MIUR.

[1] R.N. Mantegna and B. Spagnolo, Phys. Rev. Lett. 76, 563, (1996); J.E. Hirsch, B.A. Huberman and D.J. Scalapino Phys. Rev. A 25, 519, (1982).

[2] N.V. Agudov and B. Spagnolo, Phys. Rev. E 64, 035102 (R), (2001).

[3] A. N. Malakhov, A. L. Pankratov, Physica C 269, 46 (1996); D. Dan, M. C. Mahato, A. M. Jayannavar, Phys. Rev. E 60, 6421 (1999); M. Yoshimoto, Phys. Lett. A 312, 59 (2003).

[4] A.L. Pankratov and B. Spagnolo, Phys. Rev. Lett. 93, 177001 (2004); Bernardo Spagnolo, Alexander A. Dubkov, Nikolai V. Agudov, Acta Physica Polonica B, Vol. 35 (4), 1419; A. A. Dubkov, N. V. Agudov and B. Spagnolo, Phys. Rev. E 69, 061103 (2004); Evgeniya V. Pankratova, Andrey V. Polovinkin, and Bernardo Spagnolo, Physics Letters A, 344 (1), 43-50 (2005).

[5] Peter Hänggi and Fabio Marchesoni, Chaos 15, 026101/1-5 (2005).

[6] B. Spagnolo, D. Valenti, A. Fiasconaro, Math. Biosciences and Engineering 1, 185 (2004).

[7] See the special section on "Complex Systems", Science 284, 79-107 (1999); O. N. Bjornstad and B. T. Grenfell, Science 293, 638 (2001).

[8] B. B. Mandelbrot, Fractals and Scaling in Finance, Springer, New York (1997). 
[9] R.N. Mantegna and H.E. Stanley An introduction to econophysics: correlations and complexity in finance, (Cambridge University Press, Cambridge, 2000).

[10] J.P. Bouchaud and M. Potters Theory of financial risks, (Cambridge University Press, Cambridge, 2000).

[11] Lisa Borland, Jean-Philippe Bouchaud, Jean-Francois Muzy, Gilles Zumbach, The Dynamics of Financial Markets - Mandelbrot's multifractal cascades, and beyond, cond-mat/0501292 (2005); Lisa Borland and Jean-Philippe Bouchaud, On a multi-timescale statistical feedback model for volatility fluctuations, physics/0507073 (2005).

[12] J. C. Hull, Options, Futures, and Other Derivatives, Prentice-Hall, New Jersey, (1997).

[13] M.M. Dacorogna, R. Gencay, U.A. Müller, R.B. Olsen and O.V. Pictet An Introduction to High-Frequency Finance, Academic Press, New York, (2001).

[14] R. F. Engle, Econometrica 50, 987 (1982).

[15] T. Bollerslev, J. Econometrics 31, 307 (1986).

[16] S.L. Heston, Rev. Financial Studies 6, 327 (1993).

[17] H. Hull and J. White J. Finance XLII 281, (1987).

[18] J.-P. Bouchaud and R. Cont, Eur. Phys. J. B 6, 543 (1998).

[19] P. D. Ditlevsen, H. Svensmark and S. Johnsen, Nature 379, 810 (1996); P. D. Ditlevsen, Geophys. Res. Lett. 26, 1441 (1999); P. D. Ditlevsen, M. S. Kristensen, and K. K. Andersen, J. Climate 18, 2594 (2005); Xie C.W., and Mei D. C., Chin. Phys. Lett. 20 (6), 813 (2003);

[20] S. Miccichè, G. Bonanno, F. Lillo and R.N. Mantegna, Physica A 314, 756, (2002).

[21] A.A. Dragulescu and V.M. Yakovenko, Quantitative Finance 2, 443 (2002).

[22] G. Bonanno and B. Spagnolo, Fluctuation and Noise Letters 5, L325, (2005); Stochastic Models and Escape Times of Financial Markets, Mod. Probl. Stat. Phys. 4, 122 (2005).

[23] A. Christian Silva, Application of Physics to Finance and Economics: Returns, Trading Activity and Income, cond-mat/0507022 (2005).

[24] F.Black and M.Scholes, Journal of Political Economy 81, 637 (1973).

[25] R. C. Merton, Bell Journal of Economics and Management Science 4, 141 (1973).

[26] J.Cox, J.Ingersoll, and S.Ross, Econometrica 53, 385 (1985).

[27] P. Chalasani and S. Jha, Steven Shreve: Stochastic Calculus and Finance, http://www.stat.berkeley.edu/users/evans/shreve.pdf.

[28] P. Hänggi, P. Talkner and M. Borkovec, Rev. Mod. Phys. 62, 251 (1990); E. Pollak and P. 
Talkner, Chaos 15, 026116 (2005).

[29] C. W. Gardiner Handbook of stochastic methods for physics, chemistry and the natural sciences, Springer, Berlin, 1993.

[30] A. Fiasconaro, D. Valenti, B. Spagnolo, Physica A 325, 136 (2003); A. Fiasconaro, B. Spagnolo, S. Boccaletti, Phys. Rev. E72, 061110(5) (2005).

[31] J.P. Fouque, G. Papanicolau and K.R. Sircar Derivatives in financial markets with stochastic volatility, (Cambridge University Press, Cambridge, 2000).

[32] W. H. Press, S.A. Teukolsky, W. T. Vetterling, B.P. Flannery, Numerical Recipes, (Cambridge University Press, Cambridge, 1992).

[33] F. Lillo and R. N. Mantegna, Eur. Phys. J. B 15, 603 (2000).

[34] F. Lillo and R. N. Mantegna, Phys. Rev. E 62, 6126 (2000).

[35] G. Bonanno, G. Caldarelli, F. Lillo, and Rosario N. Mantegna, Phys. Rev. E 68, 046130 (2003).

[36] G. Bonanno, G. Caldarelli, F. Lillo, S. Miccichè, N. Vandewalle, and R. N. Mantegna, Eur. Phys. J. B 38, 363 (2004). 